\title{
PRESELECCIÓN DE GERMOPLASMA DE MACROPTILIUM LATHYROIDES (L.) URB. CON TOLERANCIA AL SOMBREADO
}

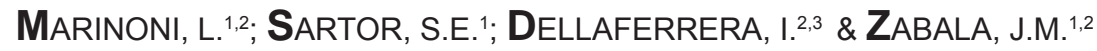

\begin{abstract}
RESUMEN
El estudio de la respuesta al sombreado en especies forrajeras nativas es una estrategia de premejoramiento útil para la selección de los materiales adaptados a los sistemas productivos silvopastoriles, predominantes en la ganadería de cría bovina de Argentina. En el presente estudio se evaluaron caracteres agronómicos y ecofisiológicos en entradas de Macroptilium lathyroides del banco de germoplasma "Ing. Agr. José M. Alonso" de la UNL, en condiciones naturales y artificiales de luz y sombra. La respuesta general estuvo asociada a una estrategia de escape al sombreado, donde las plantas elongaron más los tallos y produjeron mayor área foliar en perjuicio de la biomasa radical y foliar. Sin embargo, se identificaron entradas cuya biomasa de tallo y raíz no sufrieron modificaciones significativas bajo sombra. No obstante, la conductividad estomática y la tasa fotosintética se vieron afectadas en todas las entradas en condiciones de sombra, independientemente de la biomasa relativa al testigo.
\end{abstract}

Palabras clave: Biomasa, caracteres ecofisiológicos, escape al sombreado, recursos fitogenéticos nativos, silvopastoril.

1.- Programa de Documentación, Conservación y Valoración de la Flora Nativa. Facultad de Ciencias Agrarias. Kreder 2805.3080HOF, Esperanza, Santa Fe, Argentina.Email: lmarinoni@fca.unl.edu.ar 2.- Consejo Nacional de Investigaciones Científicas y Técnicas (CONICET), Ciudad Autónoma de Buenos Aires, Argentina.

3.- Cátedra de Cultivos Extensivos, Facultad de Ciencias Agrarias, Universidad Nacional del Litoral. Kreder 2805. 3080HOF, Esperanza, Santa Fe, Argentina.

Manuscrito recibido el 22 de abril de 2020 y aceptado para su publicación el 31 de agosto de 2020.

Marinoni, L.; Sartor, S. E.; Dellaferrera, I.; Zabala, J.M.. G. Preselección de germoplasma de Macroptilium lathyroides (L.) Urb. con tolerancia al sombreado. FAVE - Ciencias Agrarias 20 (1): 229-245.

CC BY-NC-SA 4.0 


\begin{abstract}
Preselection of germplasm of Macroptilium lathyroides (L.) Urb. with shade-tolerance.

Studies of shade effects on native forage species support pre-breeding process and are useful to establish selection criteria in plant breeding programs for the agroforestry systems prevailing in the Argentine livestock production. In the present study, ecophysiological and agronomic traits were evaluated in accessions of Macroptilium lathyroides belonging to the germplasm bank "Ing. Agr. José M. Alonso" of the UNL, under both natural and artificial light conditions. The general response has been related to a shade-avoidance strategy, where plants showed longer stems and greater leaf area in detriment of root and leaf biomass. Even so, accessions without a significant stem and root biomass reduction under shading were identified. However, stomatal conductance and photosynthetic rate were affected on all accessions subject to shade, independently of the produced biomass relative to control.
\end{abstract}

Key words: Biomass, ecophysiological traits, native plant genetic resources, shade-avoidance, agroforestry.

\section{INTRODUCCIÓN}

La producción ganadera extensiva del centro-norte de Argentina se sustenta, principalmente, de los pastizales naturales (Anderson et al., 2011). Parte de estos pastizales ocurren en sectores de bosques, donde los planteos silvopastoriles, fundamentalmente para ganadería de cría, es la actividad productiva dominante. Dado que la mayoría de estos bosques se encuadran en la Ley 26.331 (Ley de Presupuestos Mínimos de Bosques Nativos), la explotación de los mismos debe ir acompañada por un uso sustentable. Los planes incluyen manejos silvopastoriles y, en algunos casos, se plantea la introducción de forrajeras. En estos últimos, el problema radica en que son escasas las especies disponibles para estos planteos y la mayoría son especies megatérmicas que no se adaptan a la intersiembra en los pastizales (Pensiero et al., 2017). Una alternativa para mejorar la pro- ductividad de los sistemas ganaderos es la introducción a cultivo de forrajeras nativas, en particular leguminosas, por su contenido de proteína cruda y digestibilidad de la materia seca y la capacidad de mejorar el suelo vía fijación biológica de nitrógeno. Esto se ha comprobado en ambientes tropicales y subtropicales del mundo, donde la adopción de especies leguminosas nativas o introducidas aumentó la disponibilidad de nitrógeno edáfico y la calidad de la dieta animal (Congdon y Addison, 2003; Nichols et al., 2007).

Dentro de las leguminosas forrajeras nativas que potencialmente podrían utilizarse en estos sistemas se destacan especies del género Macroptilium (Benth.) Urb. (Fabaceae), las cuales son herbáceas anuales o perennes y de ciclo primavera-estivo-otoñal (Covas, 1978; Nichols et al., 2007). Macroptilium lathyroides es una especie anual o bianual nativa de América; en Argentina se la encuentra en las regiones fito- 
geográficas Paranaense, Yungas y Chaqueña (Juarez et al., 1987; Perez et al., 1999). Es una especie muy palatable y con buena resiembra, existiendo un cultivar liberado en Australia denominado "Murray" (Cameron, 1985).

En el banco de germoplasma "Ing. Agr. José M. Alonso" de la Universidad Nacional del Litoral (UNL), se conservan entradas únicas de especies nativas de interés forrajero, y particularmente, de especies del género Macroptilium. Estudios previos que han tomado como base el germoplasma disponible del banco (Zabala et al., 2015), han resultado de utilidad para los programas de mejora a partir de los cuales se ha desarrollado el cultivar "Mancebo" de $M$. lathyroides (INaSe, 2019).

Ante la necesidad de materiales mejorados que produzcan forraje en planteos silvopastoriles, muy comunes en el centro-norte de Argentina (Chiossone, 2006; Santangelo y Gil, 2016), es de suma importancia evaluar la tolerancia a condiciones de sombreado en este tipo de especies. Sin embargo, esto ha sido poco explorado en nuestro país, particularmente en especies nativas con potencial forrajero. Las plantas poseen diversas estrategias para crecer en ambientes sombreados (Addison, 2003; Congdon y Addison, 2003; Gommers et al., 2013). Por un lado, está el escape al sombreado, donde las modificaciones más frecuentes se asocian a la elongación de tallos a expensas de la producción de hojas y raíz, incremento del área foliar específica, retraso de la floración, entre otras. Por otro lado, está la tolerancia al sombreado, donde plantas tolerantes no sufren modificaciones significativas en la producción de biomasa en ambientes de baja luz y las características ecofisiológicas (contenido de clorofila, tasa fotosintética) no se ven significativamente afectadas. A través de la evaluación de tales caracteres agronómicos y ecofisiológicos, Congdon y Addison (2003) identificaron leguminosas promisorias para sistemas silvopastoriles en ambientes tropicales de Australia.

Uno de los objetivos del PRODOCOVA, en particular del banco de germoplasma de la UNL es realizar actividades de premejoramiento, las cuales comprenden diferentes caracterizaciones para identificar genes y genotipos de interés agronómico que luego puedan ser introducidos en programas de mejora genética (Nass, 2011; Sharma et al., 2017). Por este motivo se comenzó un programa para identificar leguminosas forrajeras que puedan ser utilizadas para la intersiembra en pastizales naturales en general y en particular en planteos silvopastoriles. Por lo expuesto anteriormente resulta de gran importancia en este trabajo evaluar los caracteres productivos y ecofisiológicos al estado vegetativo, en las entradas de $M$. lathyroides disponibles en el banco de germoplasma "Ing. Agr. José M. Alonso" bajo condiciones de sombreado.

\section{MATERIALES Y MÉTODOS}

\section{Experimento 1}

Se evaluaron 27 entradas de Macroptilium lathyroides del banco de germoplasma "Ing. Agr. José M. Alonso" de la UNL (Tabla 1). El ensayo se realizó en cámara de crecimiento con fotoperíodo de 12 hs a temperaturas de 25 y $18{ }^{\circ} \mathrm{C}$ durante el día y la noche, respectivamente, e iluminación con lámparas led con una densidad de flujo de fotones fotosintéticos de $640 \mu \mathrm{mol} . \mathrm{m}^{-2} \cdot \mathrm{s}^{-1}$. Estas condiciones se utilizaron como control. Para el tratamiento de sombreado, se 
L. Marinoni et al.

Tabla 1: Entradas de la colección de Macroptilium lathyroides del banco de germoplasma "Ing. Agr. José M. Alonso" de la UNL al momento de la evaluación en el presente estudio.

Table 1: Accessions of Macroptilium lathyroides collection of the germplasm bank "Ing. Agr. José M. Alonso" belonging to the UNL at the moment of present study performing.

\begin{tabular}{|c|c|c|c|c|}
\hline ID entrada & Latitud & Longitud & Origen & Observaciones \\
\hline 1 & -26.593227 & -60.642431 & Argentina (Chaco) & AusTRCF $53960^{*}$ \\
\hline 2 & \multicolumn{3}{|c|}{ CV Murray } & AusTRCF320971* \\
\hline 3 & -21.350117 & -61.524075 & Paraguay & AusTRCF $319166^{*}$ \\
\hline 5 & -21.743171 & -60.954071 & Paraguay & AusTRCF 52117* \\
\hline 7 & -12.492289 & -41.779343 & Brasil & AusTRCF $49797^{*}$ \\
\hline 8 & -25.615563 & -63.268 & Argentina (Chaco) & AusTRCF $319490^{*}$ \\
\hline 10 & 22.808721 & -80.071109 & Cuba & AusTRCF $91965^{*}$ \\
\hline 11 & \multicolumn{3}{|c|}{ Sin datos } & AusTRFC $39095^{*}$ \\
\hline 13 & -25.116886 & -64.124733 & Argentina (Salta) & AusTRCF 92586* \\
\hline 14 & -23.128463 & -64.321699 & Argentina (Salta) & AusTRCF $78441^{*}$ \\
\hline 15 & -22.167951 & -60.071414 & Paraguay & AusTRCF $319918^{*}$ \\
\hline 16 & -23.656307 & -64.083282 & Argentina (Salta) & AusTRCF $319489^{*}$ \\
\hline 17 & -20.297124 & -40.29525 & Brasil & AusTRCF 49799* \\
\hline 18 & -24.964248 & -63.791828 & Argentina (Salta) & AusTRCF $81304^{*}$ \\
\hline 19 & -22.883959 & -43.115922 & Brasil & AusTRCF $49778^{*}$ \\
\hline 20 & -26.047901 & -59.941533 & Argentina (Chaco) & AusTRCF $319491^{*}$ \\
\hline 21 & -30.107875 & -51.323476 & Brasil & AusTRCF $34903^{*}$ \\
\hline 22 & -24.89518 & -65.487204 & Argentina (Salta) & AusTRCF 92589* \\
\hline 140 & -28.516603 & -59.133307 & Argentina (Santa Fe) & ARG1188-140** \\
\hline 372 & -26.11097222 & -58.48711111 & Argentina (Formosa) & ARG1188-372** \\
\hline 485 & -28.76388 & -59.60125 & Argentina (Santa Fe) & ARG1188-485** \\
\hline 500 & -30.43394 & -59.94751 & Argentina (Santa Fe) & ARG1188-500** \\
\hline 523 & -26.81938 & -58.58442 & Argentina (Chaco) & ARG1188-523** \\
\hline 533 & -24.88061 & -59.32796 & Argentina (Formosa) & ARG1188-533** \\
\hline 537 & -23.51598 & -62.7939 & Argentina (Salta) & ARG1188-537** \\
\hline 556 & -8.514306 & -35.009191 & Brasil & ARG1188-556** \\
\hline 557 & -31.442992 & -60.94109 & Argentina (Santa Fe) & ARG1188-557** \\
\hline
\end{tabular}

* Entradas repatriadas por el Tropical Crops \& Forages Collection (Dr. Peter Lawrence), Queensland Department of Primary Industries, Biloela, Australia.

** Código WIEWS (http://www.fao.org/wiews-archive/institute.htm). 
colocó una malla plástica de media sombra de $60 \%$ entre las plantas y las lámparas, obteniéndose una densidad de flujo de fotones fotosintéticos de $290 \mu \mathrm{mol} . \mathrm{m}^{-2} \cdot \mathrm{s}^{-1}$.

Semillas de las 27 entradas fueron sembradas en macetas de $300 \mathrm{~cm}^{3}$ rellenas de tierra (Marinoni et al., 2017). En cada tratamiento (control y sombreado) se analizaron cuatro macetas por entrada, considerada la unidad experimental, donde cada una contenía tres plantas que fueron utilizadas como submuestras. Todas las macetas fueron dispuestas en un diseño completamente aleatorizado. La tierra utilizada fue extraída de los primeros $10 \mathrm{~cm}$ (horizonte A) de un suelo Argiudol típico serie Esperanza. Previo a la siembra, se realizó una escarificación física a las semillas con lija $\mathrm{n}^{\circ} 80 \mathrm{du}$ rante un minuto, debido a que las semillas de esta especie poseen una cubierta seminal dura que debe romperse para una correcta germinación. El sustrato se mantuvo a capacidad de campo durante todo el ensayo.

A los 30 días de iniciado el ensayo se extrajeron las plantas y se analizaron las siguientes variables: biomasa seca de raíz (g), tallo (g), hoja (g), biomasa total (g), relación biomasa de tallo/biomasa de hoja y raíz, área foliar $\left(\mathrm{cm}^{2}\right)$, área foliar específica ( $\mathrm{cm} 2 / \mathrm{g})$, longitud de tallo $(\mathrm{cm})$, longitud de la raíz principal $(\mathrm{cm})$, y relación longitud de tallo/longitud radical. Las muestras fueron secadas en estufa con circulación forzada de aire a $50{ }^{\circ} \mathrm{C}$ durante una semana y luego pesadas con balanza de precisión $(0,001$ g). Para las mediciones de área y longitud, las plantas fueron escaneadas con escala y las mediciones realizadas con el programa ImageJ (Rasband, 1997).

Se realizó un Análisis de la Varianza [(ANVA) $\mathrm{p}<0,05]$ para analizar las diferencias estadísticas para cada una de las variables evaluadas para un diseño factorial con los siguientes factores: entradas (27), tratamientos (2) y su interacción, previa verificación de homogeneidad de varianzas y normalidad de los datos (Shapiro-Wilks modificado). Las diferencias de medias fueron analizadas mediante la prueba de Tukey $(p<0,05)$. También se analizaron las variables en el tratamiento de sombra relativas al testigo, obtenidas como el porcentaje del valor promedio obtenido en el tratamiento de sombra en relación al tratamiento testigo. Debido a que la construcción de las variables relativas al testigo se realiza utilizando las medias, no existen repeticiones, por lo que el análisis es solamente descriptivo. Adicionalmente, se evaluó la posibilidad de compensación entre variables a través de correlaciones (Pearson, $\mathrm{p}<0,05$ ) entre los valores relativos de las variables para cada entrada, Los análisis estadísticos se realizaron con el paquete estadístico InfoStat (Di Rienzo et al., 2018).

\section{Experimento 2}

Se evaluaron cuatro entradas con comportamiento contrastante en la producción de biomasa en el Experimento 1, dos entradas con mayor biomasa relativa (ID 22, 557) y dos entradas con menor biomasa relativa (ID 1, 10). Las dos entradas con mayor biomasa relativa fueron adicionalmente selectas en función de estudios previos por su persistencia a campo y producción de semillas (datos no publicados). La evaluación se llevó a cabo en el Campo Experimental "Juan Donnet". La siembra se llevó a cabo el 3 de enero de 2018, en condiciones de iluminación plena (control), a una densidad de flujo de fotones fotosintéticos de 1800 $\mu \mathrm{mol} . \mathrm{m}^{-2} . \mathrm{s}^{-1}$ y en condiciones de sombreado, con malla plástica de media sombra 
comercial $(60 \%)$ lo que resultó en una densidad de flujo de fotones fotosintéticos de $400 \mu \mathrm{mol} . \mathrm{m}^{-2} \cdot \mathrm{s}^{-1}$. Se realizó un diseño completamente aleatorizado con cuatro repeticiones por entrada, las cuales consistían en líneas de $1 \mathrm{~m}$ donde se sembraron 50 semillas por metro lineal, cada línea separada un metro entre sí. Luego de emergidas las plántulas, se efectuó un raleo para dejar cinco plantas por línea, distanciadas a 20 $\mathrm{cm}$ entre sí, para que la competencia entre ellas fuera mínima. Estas plantas fueron utilizadas como submuestras de cada unidad experimental (línea).

A los 60 días de la emergencia, se midieron en las plantas los siguientes parámetros productivos y ecofisiológicos: biomasa seca aérea $(\mathrm{g})$, biomasa seca radical $(\mathrm{g})$, relación entre biomasa de tallo/radical, índice de verdor, conductancia estomática (mmol.m $\left.{ }^{2} \cdot \mathrm{s}^{-1}\right)$, tasa de fotosíntesis neta $\left(\mu \mathrm{mol} . \mathrm{m}^{-}\right.$ $\left.{ }^{2} \cdot \mathrm{s}^{-1}\right)$, concentración interna de $\mathrm{CO}_{2}(\mathrm{ppm})$ $\mathrm{y}$ tasa de transpiración $\left(\mathrm{mmol} \cdot \mathrm{m}^{-2} \cdot \mathrm{s}^{-1}\right)$. El índice de verdor se registró con el equipo Minolta SPAD-502® mientras que las restantes se utilizó el equipo CIRAS-2®. Para el peso seco, las muestras fueron puestas en estufa con circulación forzada de aire a 50 ${ }^{\circ} \mathrm{C}$ durante una semana y luego pesadas con balanza de precisión $(0,001 \mathrm{~g})$.

Se realizó un análisis estadístico a través de un ANVA factorial para evaluar los efectos de los tratamientos (2), entradas (4) y su interacción, en los parámetros medidos. Previamente se verificó la homogeneidad de varianzas y normalidad de los datos (Shapiro-Wilks modificado). Las diferencias de medias fueron analizadas mediante la prueba de Tukey $(\mathrm{p}<0,05)$. Se evaluaron las variables relativas al testigo para una mejor comprensión del efecto del sombrado. Los análisis estadísticos se realizaron con el paquete estadístico InfoStat (Di Rienzo et al., 2018).

\section{RESULTADOS}

\section{Experimento 1}

Se hallaron diferencias significativas entre entradas y tratamientos para todas las variables analizadas. Sólo el área foliar específica, la longitud de tallo y la relación longitud de tallo/longitud radical mostraron interacción entre factores (ver p-valores en Tabla Suplementaria 1). Todas las entradas en condiciones de sombra presentaron raíces más cortas, menor biomasa foliar, radical, de tallo y total (Tabla 2). Los valores relativos al testigo indican que la reducción más notable fue la de biomasa seca radical, con una reducción del $57 \%$ en promedio para todas las entradas en condiciones de sombreado. Contrariamente, el área foliar, el área foliar específica, la longitud de tallo, la relación biomasa de tallo/biomasa de hojas y raíz y la relación longitud de tallo/ longitud radical, aumentaron en condiciones de sombreado (Tabla 2). El área foliar específica fue el parámetro de mayor aumento $(156 \%)$.

La respuesta de las diferentes entradas para los parámetros medidos fue variable (Figura 1 y 2). Sin embargo, al evaluar la producción de biomasa en condiciones de sombreado respecto al testigo, hay entradas que muestran una escasa modificación para la mayoría de los rasgos. Los códigos de 
Tabla 2. Valores promedios (desviación estándar) para el tratamiento testigo y de sombreado, y valores en condiciones de sombreado relativos al testigo, para cada una de las variables analizadas en el Experimento 1. Letras diferentes indican diferencias estadísticas significativas (Tukey, $p<0,05$ ).

Table 2: Mean values (standard deviation) for control and shade treatments, and values under shade conditions relative to control, for each variable analysed in the Experiment 1. Different letters shown significant statistical differences (Tukey, $p<0,05$ ).

\begin{tabular}{|l|r|l|l|r|l|l|c|}
\hline \multicolumn{1}{|c|}{ VariablelTratamiento } & \multicolumn{3}{|c|}{ Testigo } & \multicolumn{3}{c|}{ Sombreado } & Valor relativo \\
\hline Área foliar (cm2) & 8.0 & $(2.2)$ & $a$ & 8.7 & $(2.6)$ & $b$ & 1.08 \\
\hline Área foliar específica (cm2/g) & 57.9 & $(16.9)$ & $a$ & 90.3 & $(38.3)$ & $b$ & 1.56 \\
\hline Longitud porción aérea (cm) & 14.2 & $(5.1)$ & $a$ & 18.5 & $(6.9)$ & $b$ & 1.30 \\
\hline Longitud radical (cm) & 15.1 & $(1.9)$ & $b$ & 13.1 & $(2.4)$ & $a$ & 0.86 \\
\hline Biomasa foliar (g) & 0.65 & $(0.24)$ & $b$ & 0.44 & $(0.20)$ & $a$ & 0.68 \\
\hline Biomasa radical (g) & 0.51 & $(0.21)$ & $b$ & 0.22 & $(0.15)$ & $a$ & 0.43 \\
\hline Biomasa de tallo (g) & 0.42 & $(0.13)$ & $b$ & 0.31 & $(0.15)$ & $a$ & 0.74 \\
\hline Biomasa total (g) & 1.59 & $(0.51)$ & $b$ & 0.98 & $(0.47)$ & $a$ & 0.62 \\
\hline Biomasa de tallo/biomasa foliar y radical & 0.39 & $(0.12)$ & $a$ & 0.50 & $(0.14)$ & $b$ & 1.28 \\
\hline Longitud porción aérea/longitud radical & 0.97 & $(0.43)$ & $a$ & 1.44 & $(0.56)$ & $b$ & 1.48 \\
\hline
\end{tabular}

identificación de estas entradas son 22, 140, $485,523,556,557$. Algunas de estas entradas también mantuvieron valores cercanos a las condiciones testigos para área foliar y área foliar específica $(140,485)$, longitud de tallo $(22,140,485)$ y longitud radical $(22,140,557)$.

El análisis de correlaciones entre valores relativos reveló una correlación significativa y positiva $(r<0,5)$ entre área foliar y las variables de biomasa (foliar, radical y de tallos), mientras que ninguna correlación se halló para el área foliar específica. La longitud radical relativa se correlacionó significativamente y positivamente $(\mathrm{r}=0,46)$ con la biomasa radical $y$, negativamente con las relaciones longitud de tallo/longitud radical y biomasa de tallo/biomasa foliar+radical $(\mathrm{r}=-0,46 ; \mathrm{r}=-0,42$, respectivamente). La longitud de tallo relativa se correlacionó significativa y positivamente con la biomasa de tallo $(\mathrm{r}=0,61)$, biomasa total $(\mathrm{r}=0,42)$ y la relación biomasa de tallo/ biomasa foliar+radical $(r=0,64)$. Los parámetros que evalúan relaciones entre variables mantuvieron correlación significativa y positiva $(\mathrm{r}=0,78)$, probablemente debido a las correlaciones entre sus componentes. Las variables de biomasa mantuvieron una alta correlación entre sí $(r>0,76)$. La matriz de correlaciones puede consultarse en Material Suplementario.

\section{Experimento 2}

En los caracteres productivos, se observaron diferencias significativas entre materiales y tratamientos e interacción para la biomasa de tallo y raíz, mientras que la relación biomasa de tallo/radical no mostró diferencias para ninguno de los factores (ver p-valores en Material Suplementario). Tanto la biomasa de raíz como la biomasa de tallo se redujeron significativamente en todos los materiales, siendo la entrada 22 la 


\section{Marinoni et al.}

Figura 1: Valores medios absolutos (+ desvío estándar) para condiciones de sombreado (barras grises) y valores relativos al tratamiento testigo (puntos negros) para las entradas de la colección de Macroptilium lathyroides del banco de germoplasma "Ing. Agr. José M. Alonso" de la UNL, para los siguientes parámetros: a) área foliar, b) área foliar específica, c) longitud de tallo, d) longitud radical y e) relación entre la longitud de tallo y longitud radical (LT/LR). DMS: diferencia mínima significativa (Tukey, $p<0,05$ ).

Figure 1: Absolute average values (plus standard deviation) for shade treatment (grey bars) and values relative to control values (puntos negros), for the accessions of Macroptilium lathyroides collection belonging to the germplasm bank "Ing. Agr. José M. Alonso", for the next traits: a) leaf area, b) specific leaf area, c) stem length, d) root length and e) stem/root length ratio (LT/LR). D: least significant difference (Tukey, $p<0,05$ ).
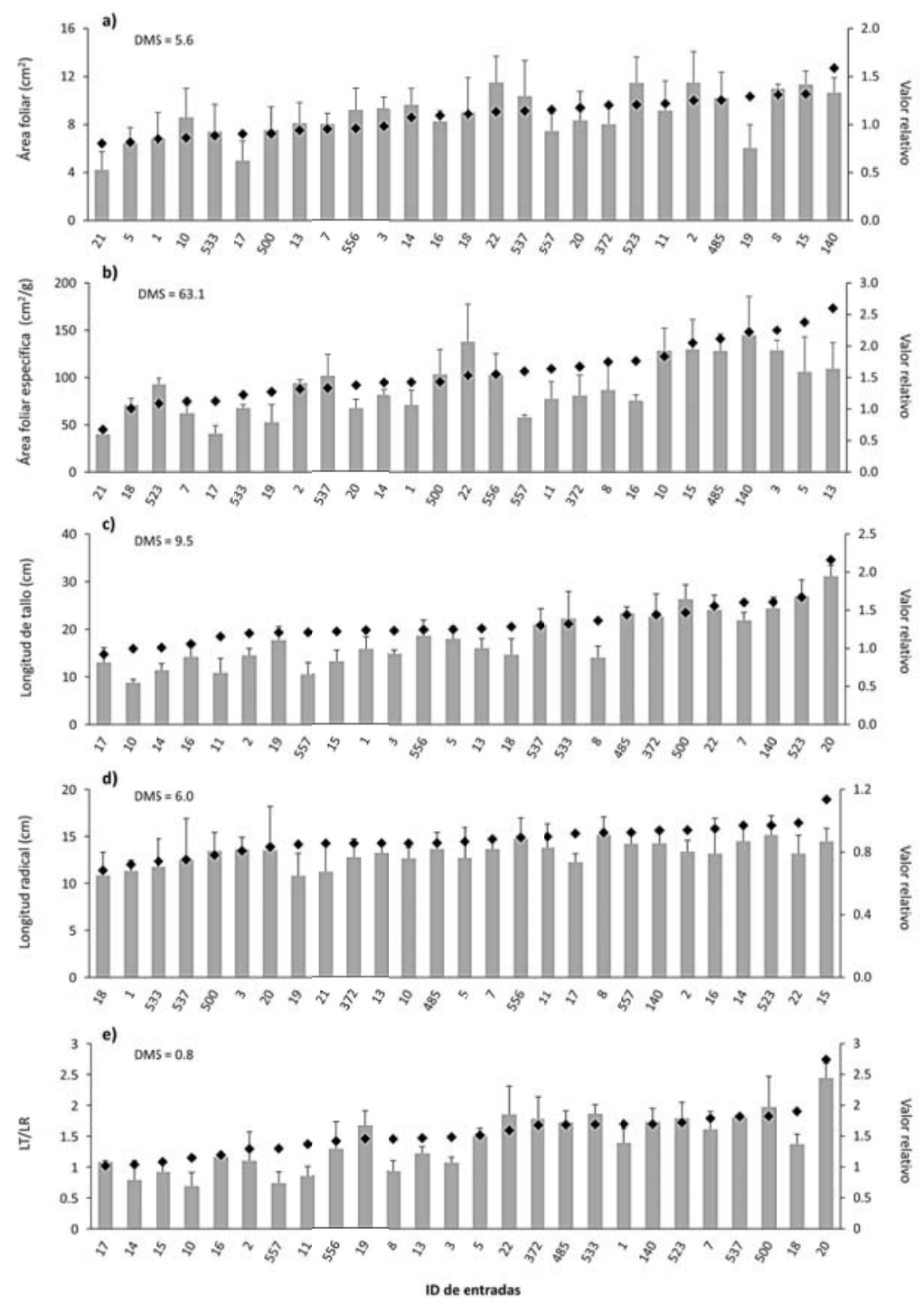
Figura 2: Valores medios absolutos (+ desvío estándar) para condiciones de sombreado (barras grises) y valores relativos al tratamiento testigo (puntos negros) para las entradas de la colección de Macroptilium lathyroides del banco de germoplasma "Ing. Agr. José M. Alonso" de la UNL, para los siguientes parámetros: a) biomasa foliar, b) biomasa radical, c) biomasa de tallos, d) biomasa total y e) relación entre la biomasa de tallos y la biomasa de raíces y hojas (BT/BR+$B F)$. DMS: diferencia mínima significativa (Tukey, $p<0,05$ ).

Figure 2: Absolute average values (plus standard deviation) for shade treatment (grey bars) and values relative to control values (puntos negros), for the accessions of Macroptilium lathyroides collection belonging to the germplasm bank "Ing. Agr. José M. Alonso", for the next traits: a) leaves biomass, $b$ ) roots biomass, $c$ ) stems biomass, $d$ ) total biomass and e) stems/roots and leaves biomass ratio $(B T / B R+B F)$. LSD: least statistical difference (Tukey, $p<0,05)$.
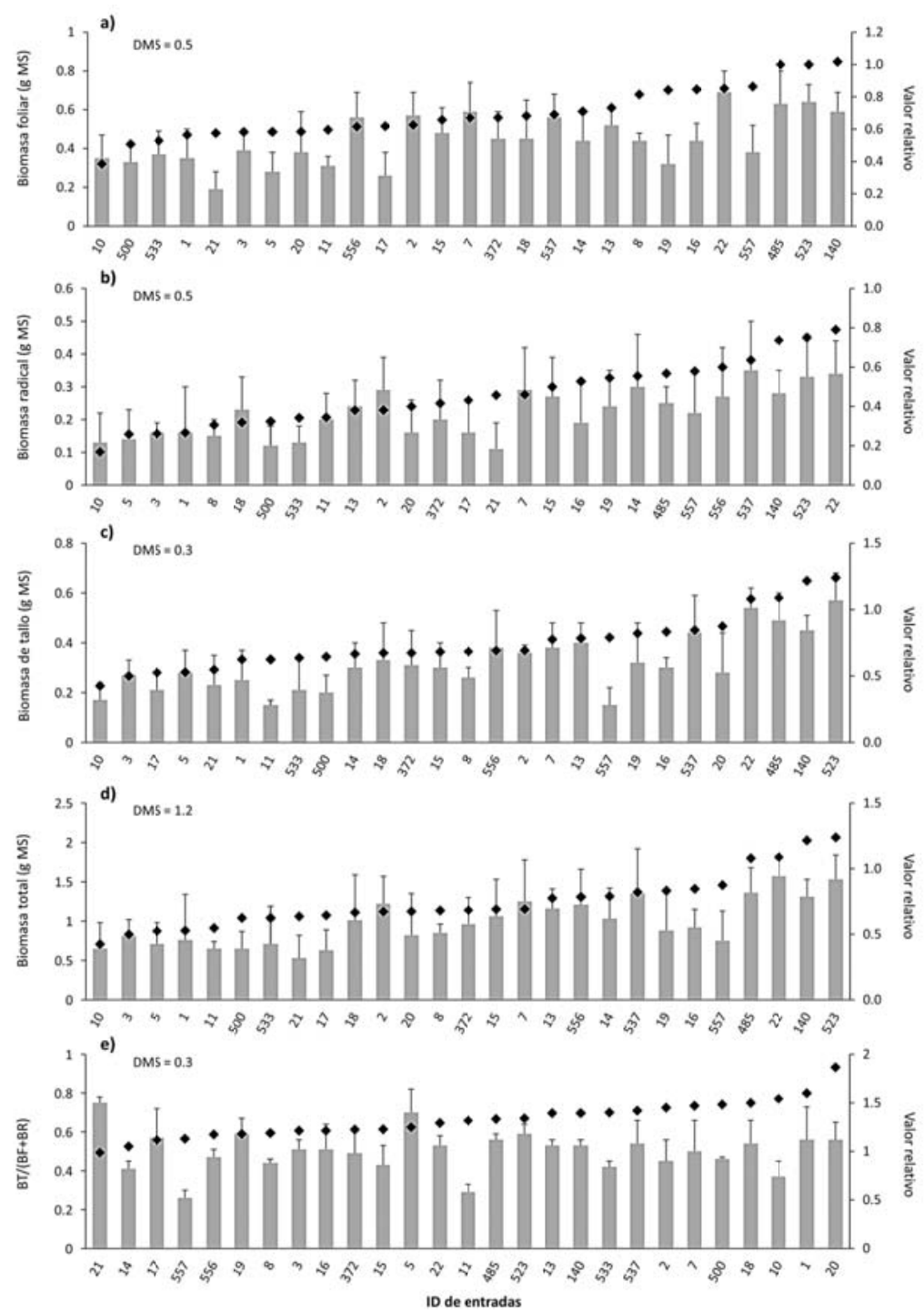

Revista FAVE - Ciencias Agrarias 20 (1) 2021 | 


\section{Marinoni et al.}

menos afectada (Figura 3). La relación entra la biomasa de tallo y la biomasa radical fue en promedio para todas las entradas de $16,9 \pm 4,2$.

Todas variables ecofisiológicas se diferenciaron sólo entre tratamientos (ver afectadas, con disminuciones del 50 y 70 p-valores en Material Suplementario). El \%, respectivamente, con relación al testigo contenido interno de $\mathrm{CO}_{2}$ fue levemente (Tabla 3).
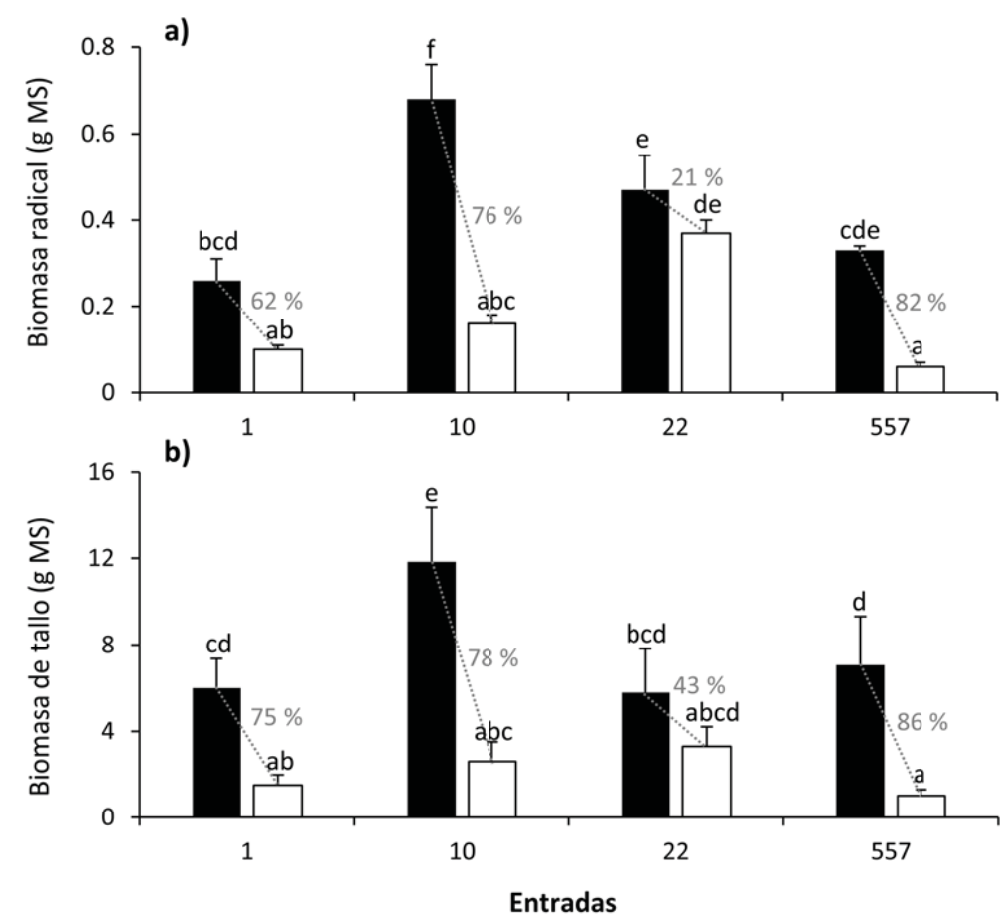

Figura 3: Valores medios absolutos (+ desvío estándar) en condiciones de sombreado (barras blancas) y testigo (barras negras) para entradas selectas de Macroptilium lathyroides, para los parámetros: a) biomasa radical y b) biomasa de tallos Letras diferentes indican diferencias significativas (Tukey, $p<0,05)$. Los valores en porcentaje revelan la disminución de los valores observados en condiciones de sombreado respecto al tratamiento testigo.

Figure 3: Absolute average values (plus standard deviation) in shade treatment (white bars) and control treatment (black bars) for selected accessions of Macroptilium lathyroides, for the next traits: a) roots biomass and b) stems biomass. Different letters denote significant differences (Tukey, $p<0,05$ ). The percentage values indicate the decrease obtained for the traits in shade conditions regarding to the control treatment values. 


\section{DISCUSIÓN}

Al inicio de todo proceso de fitomejoramiento es imprescindible contar con variabilidad para los caracteres de interés. Adicionalmente, la evaluación de la tolerancia a diversas situaciones estresantes es una herramienta de premejoramiento de gran utilidad en los planes de mejora (Panozzo y Desclaux, 2018). El presente estudio permitió detectar variabilidad entre entradas de Macroptilium lathyroides conservadas en el banco de germoplasma "Ing. Agr. José M. Alonso" de la UNL e identificar materiales promisorios para la introducción a cultivo en planteos ganaderos silvopastoriles. En términos generales, se observó que todas las entradas de la colección presentaron mayor área foliar, área foliar específica y longitud de tallos y menor longitud y biomasa radical y foliar en condiciones de sombreado respecto a lo observado en el tratamiento testigo. Adicionalmente, se observó una reducción significativa en la conductancia estomática $\mathrm{y}$, en consecuencia, en la fotosíntesis neta y la tasa de transpiración, en entradas selectas, independientemente de la producción de biomasa en condiciones de sombra.

Según Gommers y colaboradores (2013), existen dos tipos de respuesta ante condiciones de sombreado: el escape y la tolerancia. En general, las características asociadas al escape son indeseables desde el punto de vista agronómico ya que a menudo se asocian con mayor elongación de tallos, a expensas de la biomasa de raíz y hojas (Valladares y Niinemets, 2008, Gommers et al., 2013). Este fue el resultado observado en el tratamiento de sombreado, donde la longitud de tallos aumentó en todas las entradas, en promedio un $30 \%$ (respecto a la media del tratamiento testigo), contrariamente a la biomasa foliar y radical que sufrieron fuertes reducciones, algunas entradas en mayor medida que otras, las que en promedio fueron de $32 \%$ para la biomasa foliar y 57 \% para la biomasa de raíces. Las entradas identificadas con los códigos 22, $140,523,537$, y 557 fueron poco afectadas por el sombreado, ya que demostraron una

Tabla 3: Valores promedios (desviación estándar) para el tratamiento testigo y de sombreado, y valores en condiciones de sombreado relativos al testigo, para las variables ecofisiológicas evaluadas en el Experimento 2. Letras diferentes indican diferencias estadísticas significativas (Tukey, $p<0,05$ ).

Table 3: Mean values (standard deviation) for control and shade treatments, and values under shade conditions relative to control, for the ecophysiological variables analysed in the Experiment 2. Different letters shown significant statistical differences (Tukey, $p<0,05$ )

\begin{tabular}{|l|r|l|r|r|l|l|c|}
\hline \multicolumn{1}{|c|}{ VariablelTratamiento } & \multicolumn{3}{c|}{ Testigo } & \multicolumn{3}{c|}{ Sombra } & Valor relativo \\
\hline Concentración interna de $\mathrm{CO}_{2}(\mathrm{ppm})$ & 170.4 & $(38.2)$ & $a$ & 199.3 & $(18.7)$ & $b$ & 1.17 \\
\hline Conductancia estomática $\left(\mathrm{mmol} \cdot \mathrm{m}^{-2} \cdot \mathrm{s}^{-1}\right)$ & 565.6 & $(212.2)$ & $b$ & 274.6 & $(105.4)$ & $a$ & 0.49 \\
\hline Tasa de fotosíntesis neta $\left(\mu \mathrm{mol} \cdot \mathrm{m}^{-2} \cdot \mathrm{s}^{-1}\right)$ & 22.3 & $(3.3)$ & $b$ & 6.7 & $(1.5)$ & $a$ & 0.30 \\
\hline Tasa de transpiración $\left(\mathrm{mmol} \cdot \mathrm{m}^{-2} \cdot \mathrm{s}^{-1}\right)$ & 6.1 & $(1.4)$ & $b$ & 3.4 & $(1.1)$ & $a$ & 0.56 \\
\hline Índice de verdor & 49.5 & $(1.9)$ & $b$ & 37.1 & $(2.8)$ & $a$ & 0.75 \\
\hline
\end{tabular}


leve reducción en la biomasa en tales condiciones, respecto al testigo.

Por otro lado, la estrategia de tolerancia incluye una optimización en la ganancia de carbono a través del aumento del área foliar específica, de las proporciones tallo:raíz, disminución de la relación entre clorofila $a: b$, entre otras. En el presente estudio, se observó un importante aumento del área foliar específica y de la relaciones longitud de tallo:raíz y biomasa de tallo:raíz+hoja, para todas las entradas. Algunas de las entradas que no sufrieron disminución en la producción de biomasa también mantuvieron valores cercanos a las condiciones testigos para área foliar y área foliar específica (140, 485), longitud de tallo $(22,140,485)$ y longitud radical $(22,140,557)$.

Por otro lado, Addison (2003) establece otros criterios para analizar la respuesta al sombreado: tolerancia al sombreado o adaptación al sombreado. Plantas que son capaces de producir biomasa en cantidad y calidad tanto en condiciones de luz como de sombra son consideradas tolerantes (Stür, 1991; Van Sambeek et al., 2007), mientras que plantas adaptadas al sombreado, sólo lo hacen bajo sombra. Esta adaptación al sombreado es debida a ciertos rasgos adaptativos, producto de la selección natural y la evolución en ambientes con sombra. Podemos afirmar, para las entradas colectadas por nuestro banco, que ninguna fue colectada en sitios sombreados. Como ya se ha mencionado anteriormente, se pueden considerar un grupo de entradas $(22,140,523,537$, y 557) como tolerantes, ya que la producción de biomasa se vio poco o nulamente afectada en condiciones de sombra. De las entradas seleccionadas para el ensayo a campo, sólo la entrada 22 no se vio significativamente afectada en condiciones de sombra, lo que la convierte en un material valioso con fines de mejoramiento para planteos silvopastoriles. En interesante destacar que las entradas 22 y 557 poseen tallos volubles, con guías que pueden alcanzar varios metros de longitud (datos no mostrados). A futuro es necesario profundizar los estudios ecofisiológicos a fin de determinar si son útiles para planteos silvopastoriles.

Es importante destacar que, aunque los rasgos fisiológicos pueden determinar la capacidad adaptativa o la tolerancia al sombreado, por sí solos no brindan información suficiente sobre el potencial forrajero de una especie vegetal. Estos rasgos deben ir siempre acompañados de una evaluación agronómica de los materiales vegetales (Addison, 2003). Esto se vio reflejado en el presente estudio, donde los caracteres fisiológicos destacaron diferencias frente a condiciones de disponibilidad de luz, aunque no permitieron establecer diferencias entre materiales, que si se visualizó en la producción de biomasa. Es probable que exista una estrategia de compensación entre caracteres morfológicos y anatómicos que repercuta en la tasa de fotosíntesis neta de los materiales. Sin embargo, serán necesario nuevos estudios con los materiales selectos para lograr resultados más concluyentes y demostrar relaciones entre rasgos que determinen la respuesta al sombreado.

La tolerancia a la sombra no es un valor absoluto de la capacidad mínima de luz requerida por una especie determinada, sino un concepto relativo, cuyo significado depende del contexto ecológico específico (Stür, 1991; Valladares and Niinemets, 2008). La duración de la temporada de crecimiento y la concurrencia de tensiones pueden alterar dramáticamente la capaci- 
dad de tolerancia de una especie determinada, por lo que resultan imprescindibles pruebas a campo, durante períodos más prolongados, en sectores de bosques para reforzar el proceso de selección y mejora en $M$. lathyroides. En este sentido, el área foliar específica, la longitud de tallo y biomasa aérea son caracteres que explicaron diferencias en la respuesta al sombreado en el presente estudio que resultarán de gran utilidad en futuros ensayos. Adicionalmente, estos rasgos se relacionaron con caracteres de biomasa y longitud radical los que, a campo, pueden resultar difíciles de evaluar. En este sentido, el ensayo en condiciones controladas fue de gran utilidad para detectar variabilidad y los rasgos potencialmente útiles en el proceso de selección para tolerancia al sombreado.

Numerosos trabajos han demostrado la tolerancia al sombreado de leguminosas nativas forrajeras demostrando su potencial para planteos silvospastoriles. Estos estudios se han llevado a cabo particularmente para ambientes subtropicales y tropicales, donde se valoran especies de los géneros Desmodium, Trifolium, Centrosema, Arachis, Aeschynomene, Stylosanthes (Stür, 1991; Addison, 2003; Congdon y Addison, 2003; Van Sambeek et al., 2007; Pang et al., 2017).

Congdon y Addison (2003) destacan a $M$. lathyroides como una de las 16 especies consideradas como tolerantes en estado vegetativo (evaluaron 35 especies diferentes) de acuerdo a su criterio de discriminación. En este estudio se analizó un único material, el cultivar "Murray". En nuestro trabajo se analizó dicho cultivar en el ensayo 1 (ID entrada 2), mostrando un comportamiento intermedio en términos productivos. Esto demuestra el potencial del germoplasma conservado en el banco de germoplasma de la UNL para tolerancia a la sombra.
En otros ensayos, el cultivar "Siratro" de Macroptilium atropurpureum (L.) Urb. ha sido más valorado por su potencial forrajero en ambientes sombreados (Richards, 1988; Stür, 1991). Es interesante destacar que $M$. atropurpureum, si bien es perenne, tiene el mismo hábito de crecimiento que las entradas 22 y 557, es decir, posee tallos volubles. Este hábito podría ser una ventaja en ambientes sombreados, ya sea en competencia con otras especies como gramíneas o en sistemas silvopastoriles. Por otro lado, el cultivar "Murray" tiene hábito erecto, por lo que estudios posteriores podrán permitir mayores inferencias sobre el efecto del hábito de crecimiento y la tolerancia a la sombra.

\section{CONCLUSIÓN}

El presente estudio permitió generar información acerca del comportamiento bajo sombra de una forrajera nativa de Argentina para su utilización en planteos silvopastoriles. Se halló variabilidad en la respuesta al sombreado en Macroptilium lathyroides; en general, detectando entradas tolerantes según el criterio de Addison (2003), con una estrategia de escape al sombreado de acuerdo a la clasificación de Gommers et al. (2013).

La información generada en este trabajo es importante en términos de identificación de entradas con respuesta contrastante al sombreado en $M$. lathyroides. En este sentido, el ensayo en ambiente controlado permitió detectar variabilidad en un gran número de entradas para los rasgos de interés e identificar caracteres potencialmente útiles en el proceso de mejora para ambientes sombreados como el área foliar específica, la longitud de tallo y la biomasa aérea. La in- 
formación generada en este trabajo será aplicada a ensayos a campo adicionales, evaluando entradas contrastantes identificadas en condiciones reales de sombreado, bajo bosque, y evaluando caracteres productivos (productividad, fijación biológica de nitrógeno, producción de semillas) y fisiológicos durante todo el ciclo productivo de la pastura para determinar la factibilidad de uso de esta especie en planteos silvopastoriles.

\section{AGRADECIMIENTOS}

El presente trabajo fue financiado con el proyecto PICTO Bosques 2014-011 (Res. $\mathrm{N}^{\circ}$ 716/15. Período 2016-2019) "Forrajeras nativas para sistemas silvopastoriles del Parque Chaqueño argentino", bajo la dirección del Dr. José Pensiero y con del Programa de Documentación, Conservación y Valoración de la Flora Nativa (PRODOCOVA) de la FCA-UNL.

\section{BIBLIOGRAFÍA}

Addison H. 2003. Shade tolerance of tropical forage legumes for use in agroforestry systems. Tesis Doctoral. James Cook University.

Anderson DL, Bonvissuto GL, Brizuela MA, Chiossone G, Cibils AF, Cid MS, ... Oliva GE. 2011. Perspectives on Rangeland Management Education and Research in Argentina. Rangelands. 33(1):2-12.

Cameron DG. 1985. Tropical and subtropical legumes 8: Phasey bean (Macroptilium lathyroides). The predecessor of Siratro. Queensland Agric J. 111:211-214.

Chiossone G. 2006. Sistemas de producción ganaderos del noreste argentino. Situación actual y propuestas tecnológicas para mejorar su productividad. p. 120-137. X Seminario de pastos y forrajes. Universidad del Zulia- Luz, FCV Maracaibo, Venezuela. 20-22 de abril de 2006. https://www.produccionanimal.com.ar/ informacion-tecnica/origenesevolucion-y-estadisticas-de-la-ganaderia/65- Guillermo-Chiossone.pdf. (Consulta 30/03/2020).

Congdon B, Addison H. 2003. Optimising nutrition for productive and sustainable farm forestry systems: pasture legumes under shade. Rural and Industries Research Development Corporation (RIRDC) Publication $\mathrm{N}^{\circ}$ 03/113. 106 pp.

Convenio UNL-PEMAN. Convenio de Vinculación Tecnológica entre la Universidad Nacional del Litoral y la Empresa Oscar Peman y Asociados S.A., "Colaboración para I+D en tecnología de producción de semillas" a partir del 2014 y por el término de 3 años, cuyo objetivo es el desarrollo de un paquete tecnológico para la producción de semillas en forrajeras leguminosas nativas. Expte. UNL 617099.

Covas G. 1978. Forrajeras indígenas: Especies que requieren un plan de conservación de germoplasma. Ciencia e Investigación. 34:209-213.

Di Rienzo JA, Casanoves F, Balzarini MG, González L, Tablada M, Robledo CW. 2018. InfoStat versión 2018. Grupo InfoStat, FCA, Universidad Nacional de Córdoba, Argentina. URL http://www.InfoStat.com.ar.

Gommers CM, Visser EJ, St Onge KR, Voesenek LA, Pierik R. 2013. Shade tolerance: when growing tall is not an option. Trends Plant Sci. 18(2):65-71.

INaSe, 2019. Cultivar "Mancebo" (Macroptilium lathyroides). Catálogo Nacional de Cultivares. https://www.inase.gov.ar/consultaGestion/gestiones. (Consulta: 20/08/19)

Juarez FC, Perez, SM. 1987. El género Macroptilium (Fabaceae) en la Provincia de Salta, Argentina. Anales INTA Salta 1:31-42. 
Marinoni LDR, Zabala JM, Patiño J, Pensiero JF. 2017. Efecto de la temperatura y salinidad en la germinación y crecimiento inicial de un material naturalizado de Lotus tenuis Waldst. \& Kit. Revista FAVE - Ciencias Agrarias 16 (2): 47-59.

Nass L. 2011. Pré-melhoramento vegetal. In: Lopes MA, Fávero AP, Ferreira MAJF, Faleiro FG, Folle SM, Guimarães EP. Pré-melhoramento de plantas: estado da arte e experiências de sucesso. Brasília, DF: Embrapa Informação tecnológica: Embrapa Recursos Genéticos e Biotecnologia; Planaltina, DF: Embrapa Cerrados, p. 23-38.

Nichols PGH, Loi A, Nutt BJ, Evans PM, Craig AD, Pengelly BC, ... Ewing MA. 2007. New annual and short-lived perennial pasture legumes for Australian agriculture-15 years of revolution. Field Crop Res. 104(1-3):10-23.

Pang K, Van Sambeek JW, Navarrete-Tindall NE, Lin CH, Jose S, Garrett HE. 2019. Responses of legumes and grasses to non-, moderate, and dense shade in Missouri, USA. I. Forage yield and its species-level plasticity. Agroforest Syst. 93(1):11-24.

Panozzo A, Desclaux D. 2018. Breeding for agroforestry: is it only breeding for shade? European Agroforestry Conference-Agroforestry as sustainable Land Use, 4th. EURAF. Pp. 373-377.

Pensiero JF, Zabala JM, Marinoni L, Richard G. 2017. Recursos fitogenéticos forrajeros nativos y naturalizados (RFNyN) para suelos salinos de la región chaqueña de Argentina. En: Taleisnik E, Lavado R (Eds.). La salinidad y alcalinidad en suelos de la Argentina y su efecto sobre vegetación natural y cultivos. Prospección y manejo para su aprovechamiento productivo. Orientación Gráfica Editora, Ciudad Autónoma de Buenos Aires, Facultad de Ciencias Agropecuarias, Universidad Católica de Córdoba, Córdoba, Argentina. Pp. 373-409.

Perez SM, Camardelli MC, Juarez F, Bianchi
AR, Newman R. 1999. Geographical distribution of Macroptilium species in Argentina. Trop Grassl. 33:22-33.

Rasband WS. 1997. ImageJ, U. S. National Institutes of Health, Bethesda, Maryland, USA, https://imagej.nih.gov/ij/.

Reynolds SG. 1988. Pastures and Cattle under Coconuts. FAO Plant Production and Protection Paper 91. Food and Agricultural Organization of the United Nations, Rome, Italy.

Santangelo F, Gil F. 2016. Potencial productivo de la ganadería bovina de la provincia de Santa Fe. http://www.ipcva.com.ar/documentos/1620_1473257551_potencialproductivodelaprovinciadesantafe.pdf. (Consulta 21/03/2020).

Sharma S. 2017. Prebreeding using wild species for genetic enhancement of grain legumes at ICRISAT. Crop Sci 57: 1132-1144.

Stür WW. 1991. Screening forage species for shade tolerance - a preliminary report. En: Shelton HM, Stür WW (Eds). Forages for plantation crops. ACIAR Proceedings $\mathrm{N}^{\circ}$ 32. Pp. 58-63.

Valladares F, Niinemets Ü. 2008. Shade tolerance, a key plant feature of complex nature and consequences. Annu Rev Ecol Evol Syst. 39:237-257.

Van Sambeek JW, Navarrete-Tindall NE, Garrett HE, Lin CH, McGraw RL, Wallace DC. 2007. Ranking the shade tolerance of forty-five candidate groundcovers for agroforestry plantings. The Temperate Agroforester. 15 (4):10 p.

Zabala JM, Pensiero JF, Forni M, Sosa N, Testa M, Giavedoni J, Aiello F, Yost A, Quarin P. 2015. Valorización de los recursos fitogenéticos a través de pequeñas empresas productoras de semillas de forrajeras nativas: evaluación de algunos factores que afectan la producción de semillas en leguminosas forrajeras. En Red de Cultivos no Tradicionales de Agricultura Familiar. XVII Foro de Decanos de Facultades de Agronomía del Mercosur, Bolivia y Chile. UNER. Pp 65-73. 
L. Marinoni et al.

\section{APÉNDICE}

Material Suplementario

Tabla Suplementaria 1: Valores de probabilidad derivados del Análisis de la Varianza $(p<0,05)$ en la evaluación de caracteres ecofisiológicos y agronómicos en entradas de la colección de Macroptilium lathyroides del banco de germoplasma “Ing. Agr. José M. Alonso” sometidas a condiciones de sombreado.

\begin{tabular}{|l|c|c|c|}
\hline \multicolumn{1}{|c|}{ Fuente de variación } & Tratamiento $(\mathrm{T})$ & Entrada $(\mathrm{E})$ & $\mathrm{T}^{*} \mathrm{E}$ \\
\hline Variable & & & \\
\hline Área foliar $\left(\mathrm{cm}^{2}\right)$ & 0.0135 & $<0.0001$ & $\mathrm{NS}$ \\
\hline Área foliar específica $\left(\mathrm{cm}^{2} / \mathrm{g}\right)$ & $<0.0001$ & $<0.0001$ & 0.0001 \\
\hline Longitud porción aérea $(\mathrm{cm})$ & $<0.0001$ & $<0.0001$ & $<0.0001$ \\
\hline Longitud radical $(\mathrm{cm})$ & $<0.0001$ & 0.0145 & $\mathrm{NS}$ \\
\hline Biomasa foliar $(\mathrm{g})$ & $<0.0001$ & $<0.0001$ & $\mathrm{NS}$ \\
\hline Biomasa radical $(\mathrm{g})$ & $<0.0001$ & 0.0066 & $\mathrm{NS}$ \\
\hline Biomasa de tallo $(\mathrm{g})$ & $<0.0001$ & $<0.0001$ & $\mathrm{NS}$ \\
\hline Biomasa total $(\mathrm{g})$ & $<0.0001$ & $<0.0001$ & $\mathrm{NS}$ \\
\hline Biomasa de tallo/biomasa foliar y radical & $<0.0001$ & $<0.000$ & $\mathrm{NS}$ \\
\hline Longitud de tallo/longitud radical & $<0.0001$ & $<0.0001$ & $<0.0001$ \\
\hline
\end{tabular}

NS: Diferencias no significativas $(p>0,05)$

Tabla Suplementaria 2: Matriz de correlación (Pearson, $p<0,05)$ entre caracteres ecofisiológicos y agronómicos evaluados en entradas de la colección de Macroptilium lathyroides del banco de germoplasma "Ing. Agr. José M. Alonso" en condiciones de sombreado.

\begin{tabular}{|c|c|c|c|c|c|c|c|c|c|c|}
\hline & Área foliar & $\begin{array}{l}\text { Área foliar } \\
\text { especifica }\end{array}$ & $\begin{array}{c}\text { Longitud } \\
\text { radical }\end{array}$ & $\begin{array}{l}\text { Longitud } \\
\text { de tallo }\end{array}$ & $\begin{array}{c}\text { Longitud de } \\
\text { tallo/Longitud } \\
\quad \text { radical }\end{array}$ & $\begin{array}{l}\text { Biomasa } \\
\text { radical }\end{array}$ & $\begin{array}{c}\text { Biomasa } \\
\text { foliar }\end{array}$ & $\begin{array}{l}\text { Biomasa } \\
\text { de tallo }\end{array}$ & $\begin{array}{c}\text { Biomasa } \\
\text { total }\end{array}$ & \begin{tabular}{|c|} 
Biomasa de \\
tallo/Biomasa \\
foliar y \\
radical \\
\end{tabular} \\
\hline Área foliar & 1.00 & 0.37 & 0.02 & 0.05 & 0.41 & 0.01 & 0.00 & 0.00 & 0.00 & 0.96 \\
\hline Área foliar específica & 0.18 & 1.00 & 0.26 & 0.77 & 0.82 & 0.53 & 0.44 & 0.78 & 0.84 & 0.99 \\
\hline Longitud radical & 0.43 & 0.22 & 1.00 & 0.81 & 0.02 & 0.01 & 0.05 & 0.17 & 0.05 & 0.03 \\
\hline Longitud de tallo & 0.38 & 0.06 & -0.05 & 1.00 & 0.00 & 0.19 & 0.14 & 0.00 & 0.03 & 0.00 \\
\hline $\begin{array}{l}\text { Longitud de } \\
\text { tallo/Longitud radical }\end{array}$ & 0.17 & -0.05 & -0.46 & 0.89 & 1.00 & 0.95 & 0.76 & 0.05 & 0.41 & 0.00 \\
\hline Biomasa radical & 0.51 & -0.13 & 0.46 & 0.26 & 0.01 & 1.00 & 0.00 & 0.00 & 0.00 & 0.17 \\
\hline Biomasa foliar & 0.69 & 0.16 & 0.38 & 0.29 & 0.06 & 0.76 & 1.00 & 0.00 & 0.00 & 0.22 \\
\hline Biomasa de tallo & 0.64 & 0.06 & 0.27 & 0.61 & 0.39 & 0.82 & 0.86 & 1.00 & 0.00 & 0.45 \\
\hline Biomasa total & 0.64 & 0.04 & 0.40 & 0.42 & 0.16 & 0.91 & 0.93 & 0.95 & 1.00 & 0.50 \\
\hline $\begin{array}{c}\text { Biomasa de } \\
\text { tallo/Biomasa foliar y } \\
\text { radical }\end{array}$ & 0.01 & 0.00 & -0.42 & 0.64 & 0.78 & -0.27 & -0.24 & 0.15 & -0.13 & 1.00 \\
\hline
\end{tabular}


Tabla Suplementaria 3: Valores de probabilidad derivados del Análisis de la Varianza $(p<0,05)$ en la evaluación de caracteres ecofisiológicos y agronómicos en materiales selectos de Macroptilium lathyroides sometidas a condiciones de sombreado.

\begin{tabular}{|l|c|c|c|}
\hline \multicolumn{1}{|c|}{ Fuente de variación } & Tratamiento $(\mathrm{T})$ & Entrada $(\mathrm{E})$ & $\mathrm{T}^{*} \mathrm{E}$ \\
\hline Variable & & & \\
\hline Concentración interna de $\mathrm{CO}_{2}(\mathrm{ppm})$ & 0.0008 & $\mathrm{NS}$ & $\mathrm{NS}$ \\
\hline Conductancia estomática $\left(\mathrm{mmol} \cdot \mathrm{m}^{-2} \cdot \mathrm{s}^{-1}\right)$ & $<0.0001$ & $\mathrm{NS}$ & $\mathrm{NS}$ \\
\hline Tasa de fotosíntesis neta $\left(\mu \mathrm{mol} \cdot \mathrm{m}^{-2} \cdot \mathrm{s}^{-1}\right)$ & $<0.0001$ & $\mathrm{NS}$ & $\mathrm{NS}$ \\
\hline Tasa de transpiración $\left(\mathrm{mmol} \cdot \mathrm{m}^{-2} \cdot \mathrm{s}^{-1}\right)$ & $<0.0001$ & $\mathrm{NS}$ & $\mathrm{NS}$ \\
\hline Índice de verdor & $<0.0001$ & $\mathrm{NS}$ & $\mathrm{NS}$ \\
\hline Biomasa seca radical $(\mathrm{g})$ & $<0.0001$ & $<0.0001$ & 0.0001 \\
\hline Biomasa seca de tallo $(\mathrm{g})$ & $<0.0001$ & 0.0001 & 0.0363 \\
\hline Biomasa de tallo/biomasa radical & $\mathrm{NS}$ & 0.0034 & $\mathrm{NS}$ \\
\hline
\end{tabular}

NS: Diferencias no significativas ( $p>0,05)$ 